\title{
Abstinência de Benzodiazepinas: Um Caso Clínico
}

\section{Benzodiazepines Withdrawal: A Case Report}

Teresa Rei ${ }^{1}$, Sérgio Miguel Martinho², Cláudia Melo³

Autor Correspondente/Corresponding Author:

Teresa Rei Silva [teresareisilva7@gmail.com]

\section{RESUMO}

As benzodiazepinas constituem uma classe de fármacos com ação ansiolítica, sedativa e hipnótica, sendo amplamente utilizadas no tratamento de perturbação da ansiedade e perturbação do sono. Não obstante a sua elevada eficácia, os efeitos adversos e o risco de dependência e tolerância a que estão associadas condicionam o seu uso de uma forma generalizada.

Relativamente à descontinuação de benzodiazepinas, tem-se verificado uma baixa adesão às orientações e insuficiente reavaliação do tratamento. Os regimes terapêuticos são mantidos indefinidamente, sem reavaliação periódica da indicação para a sua manutenção.

Descreve-se o caso clínico de uma doente de 66 anos que recorre ao serviço de urgência por agitação psicomotora, alteração comportamental e discurso incoerente após interrupção abrupta de benzodiazepina.

O presente artigo pretende relatar um episódio de síndrome de abstinência de benzodiazepinas e alertar os profissionais de saúde para as precauções a ter na desprescrição deste grupo farmacológico.

PALAVRAS-CHAVE: Benzodiazepinas/efeitos adversos; Benzodiazepinas/uso terapêutico; Desprescrições; Síndrome de Abstinência a Substâncias

1. Interna de Formação Específica em Medicina Geral e Familiar, Agrupamento de Centros de Saúde Cávado I - Braga, USF Gualtar, Braga, Portugal. 2. Interno de Formação Específica em Psiquiatria, Serviço de Psiquiatria e Saúde Mental do Centro Hospitalar de Leiria, Leiria, Portugal. 3. Assistente Graduada em Medicina Geral e Familiar, Agrupamento de Centros de Saúde Cávado I - Braga, USF Gualtar, Braga, Portugal.

${ }^{\circ}$ Autor (es) (ou seu (s) empregador (es)) 2019. Reutilização permitida de acordo com CC BY-NC. Nenhuma reutilização comercial. ${ }^{\circledR}$ Author(s) (or their employer(s)) 2019. Re-use permitted under CC BY-NC. No commercial re-use. 


\section{ABSTRACT}

Benzodiazepines are a class of drugs with anxiolytic, sedative and hypnotic action. Consequently, they are commonly used in the treatment of anxiety and sleep disorders. Notwithstanding their remarkable efficacy, adverse effects and dependency risks limit their widespread and continued use.

Regarding the discontinuation of benzodiazepines there has been a low adherence to the guidelines. Treatments are maintained indefinitely, without timely reassessment of treatment indication.

The clinical case of a 66-year-old patient observed in emergency department due to psychomotor agitation, behavioural changes and incoherent speech after discontinuation of benzodiazepine is described.

The present article aims to report an episode of benzodiazepine withdrawal syndrome and to alert health professionals to the precautions they should bear in mind when stopping drugs from this pharmacological group.

KEYWORDS: Benzodiazepines/adverse effects; Benzodiazepines/therapeutic use; Deprescriptions; Substance Withdrawal Syndrome

\section{INTRODUÇÃO}

As benzodiazepinas constituem uma classe de fármacos com propriedades ansiolítica, sedativa e hipnótica. A sua crescente prescrição nas últimas décadas tornou-as num dos grupos farmacológicos mais utilizados. ${ }^{1}$ Com efeito, Portugal encontra-se nos primeiros lugares de consumo de benzodiazepinas na Europa, facto que motivou recomendações sobre a prescrição racional desta classe farmacológica no país por parte da International Narcotic Board. ${ }^{2}$ As benzodiazepinas são um grupo farmacológico com evidência de eficácia e a sua utilidade é atestada pela presença em orientações clínicas para o tratamento de várias perturbações de ansiedade, ${ }^{3}$ bem como na orientação de perturbações do sono, desde a insónia às parassónias. ${ }^{4,5}$

Não obstante a sua eficácia, os efeitos adversos e o risco de dependência e habituação a que estão associadas condicionam o seu uso de uma forma generalizada e continuada. ${ }^{1}$ Entre os efeitos adversos que se observam mais frequentemente encontram-se a sonolência, alterações cognitivas, nomeadamente confusão e desorientação, bem como alterações motoras, com ataxia e aumento do risco de quedas e fraturas, principalmente na população idosa. ${ }^{1}$

Deste modo, é fulcral salientar que o seu uso tem indicações médicas precisas, devendo ser limitado no tempo. A Agência Europeia do Medicamento emitiu recomendações, em 1991, limitando o uso de benzodiazepinas ao tratamento da ansiedade ou da insónia graves e incapacitantes, em indivíduos sujeitos a desgaste intenso. ${ }^{6}$ Assim, o seu uso no tratamento da ansiedade e insónia ligeiras a moderadas não está recomendado. ${ }^{6}$ De acordo com as normas de prescrição de benzodiazepinas e análogos em Portugal, a duração recomendada na ansiedade patológica não deverá ultrapassar as 8 a 12 sema- nas e, na insónia, as 4 semanas, incluindo o período de descontinuação. ${ }^{6}$

Tendencialmente, o surgimento de efeitos secundários aumenta com a semivida do fármaco. Os efeitos adversos e o risco de dependência tendem, ainda, a aumentar em função da dose e da duração do tratamento. Indivíduos com antecedentes de dependência ao álcool e/ou drogas têm uma maior predisposição para a dependência de benzodiazepinas. Adicionalmente, as benzodiazepinas de semivida mais curta conduzem mais frequentemente a dependência. ${ }^{1}$

Embora a melhor estratégia seja a prevenção do abuso e da dependência de benzodiazepinas aquando da sua prescrição, a sua descontinuação deverá ser sempre considerada quando o seu uso se revela inadequado. Os desafios à desprescrição de benzodiazepinas são variados. Está recomendada a sua suspensão gradual, durante semanas a meses, associada a consultas de seguimento para reavaliação dos sintomas. A capacitação do utente, mantendo-o informado no decurso deste processo, é fundamental para o seu sucesso.

$\bigcirc$ presente artigo relata um episódio de síndrome de abstinência de benzodiazepinas e tem como propósito alertar os profissionais de saúde para as precauções a ter aquando da desprescrição deste grupo farmacológico.

\section{CASO CLÍNICO}

Doente do sexo feminino, 66 anos, casada, com 2 filhos, observada no serviço de urgência (SU) por agitação psicomotora, alteração comportamental e discurso incoerente com um dia de evolução.

Tratava-se de uma doente com antecedentes de síndrome depressiva diagnosticada há 12 anos, fibrilhação au- 
ricular, hipertensão arterial, osteoporose e síndrome de apneia obstrutiva do sono sob ventilação não-invasiva. Sem outros antecedentes psiquiátricos, nomeadamente dependência de substâncias.

De acordo com a informação veiculada, a doente estaria medicada habitualmente com sertralina 50 mg, bisoprolol 2,5 mg, apixabano 5 mg, ácido alendrónico 70 mg, e trazodona $150 \mathrm{mg}$.

Encontrava-se reformada (administrativa) e tinha como habilitações literárias o $9^{\circ}$ ano de escolaridade. Residia com o marido, inserida numa família nuclear, na fase VIII do Ciclo de Vida de Duvall, sendo totalmente independente para as atividades de vida diária.

Entrou no SU acompanhada pela filha que referia que a mãe estaria muito agitada e eufórica durante esse dia, com discurso repetitivo e de teor messiânico. A doente apresentava-se gravemente agitada, com discurso manifestando alterações do conteúdo do pensamento, sob a forma de ideação delirante místico-grandiosa, afirmando que "vai revelar o mistério do mundo, que todos vamos ser atingidos". A atenção da doente era fracamente captável, com períodos de perplexidade e interação sem objeto, intuindo-se a presença de alterações da sensoperceção. A doente não tinha qualquer crítica para os fenómenos.

Durante a permanência no SU, foi realizado estudo analítico que incluiu hemograma completo, função renal e hepática, ionograma e glicemia, análise de urina tipo II. Efetuou tomografia computorizada cranioencefálica que não revelou alterações morfológicas ou densitométricas significativas, com vias de circulação de líquido cefalorraquidiano de amplitude e morfologia normais.

Foi medicada com haloperidol 5 mg e diazepam 10 mg e pernoitou no SU a aguardar observação por Psiquiatria.

Verificou-se uma redução progressiva da agitação psicomotora. À reavaliação, encontrava-se vígil, calma e colaborante, orientada no tempo e espaço. Apresentava discurso coerente e organizado de volume e fluxo normal e mantinha bom contacto ocular. Apesar de humor subdeprimido com qualidade ansiosa, não era manifesta labilidade emocional. Não apresentava ideação suicida estruturada, alterações do pensamento ou da perceção, com comportamento adequado. $\bigcirc$ exame neurológico não mostrou quaisquer alterações ou défices significativos. A doente demonstrava crítica para o episódio, corroborando não serem congruentes com o seu comportamento habitual.

Após observação, a doente informou que tomava lorazepam 2,5 mg, há doze anos e assumiu ter suspendido abruptamente essa benzodiazepina há uma semana, apesar de ter indicação da sua médica assistente para iniciar esquema de descontinuação gradual de benzodiazepina.

Por agravamento dos níveis de ansiedade, reativos a diagnóstico recente de demência do marido e conflitos numa instituição que frequentava optou-se por realizar ajuste terapêutico com alteração da medicação antidepressiva para escitalopram 10 mg id e manteve trazodona $150 \mathrm{mg}$ (1/3 a 2/3 ao deitar).

Após exclusão de causa orgânica e mediante evolução favorável do quadro com consciência e juízo crítico para o sucedido anteriormente, optou-se por alta para ambulatório com agendamento de consulta para reavaliação.

Na reavaliação após um mês, constatou-se resolução gradual do quadro clínico, tendo a doente recuperado o seu funcionamento prévio.

\section{DISCUSSÃO}

O relato de caso descrito é ilustrativo de síndrome de abstinência de benzodiazepinas dada a relação temporal do aparecimento do quadro sintomático com a suspensão abrupta de lorazepam associada à recuperação completa e evolução favorável do episódio.?

As reações adversas do uso continuado de benzodiazepinas são dose-dependente, e relacionadas com a duração do tratamento e semivida do fármaco. Contudo, benzodiazepinas de semivida curta (< 8 horas) assumem um maior risco de adição. Os efeitos adversos são ainda potenciados pelo uso concomitante de outras substâncias psicoativas, como o álcool. ${ }^{1}$

Embora a descontinuação de benzodiazepinas possa decorrer de forma tranquila na maioria dos casos, estima-se que 20\% a 50\% dos indivíduos com consumo prolongado de benzodiazepinas venham a manifestar sintomas de abstinência, indicando a presença de dependência de substância. ${ }^{8}$

A síndrome de abstinência corresponde ao conjunto de sintomas físicos e psicológicos que surgem após descontinuação ou redução rápida da dose de uma substância (Tabela 1). ${ }^{9}$ Habitualmente inicia-se nos primeiros dois dias após redução ou interrupção de uma benzodiazepina de semivida curta ou cinco a sete dias, se de semivida longa. Se não for devidamente reconhecida, esta síndrome pode assemelhar-se ao quadro inicial que condicionou a prescrição, incentivando a sua manutenção ou mesmo o aumento da posologia. ${ }^{6}$ 
TABELA 1. Manifestações clínicas da síndrome de abstinência a benzodiazepinas.

\section{Síndrome de Abstinência a Benzodiazepinas - Manifestações Clínicas}

GERAIS

> Cefaleias

> Palpitações

> Sudorese

\section{MUSCULOESQUELÉTICAS}

, Tremor, fasciculações

, Dor, rigidez e espasmos musculares (particularmente na face e couro cabeludo)

\section{NEUROLÓGICAS}

, Tontura, light-headedness

> Parestesias, dores no pescoço e coluna

> Distúrbios visuais (visão turva, diplopia, fotofobia)

, Zumbido

> Desmaio e tontura, sensação de instabilidade

, Confusão, desorientação (pode ser intermitente) - uma causa comum de confusão em idosos

, Delirium (na ausência de hiperatividade autonómica) particularmente em idosos

> Alucinações (visuais, auditivas)

> Convulsões

\section{GASTROINTESTINAIS}

\section{> Náusea}

> Anorexia, perda ponderal

, Diarreia (pode assemelhar-se à síndrome do intestino irritável)

\section{PSICOLÓGICAS}

\section{> Insónia rebound, terrores noturnos}

> Ansiedade, ataques de pânico

> Irritabilidade, inquietação, agitação

> Sentimentos e pensamentos paranoicos

, Dificuldade de concentração e na memória

> Distorções percetivas - hipersensibilidade sensorial (luz, som, tato, paladar), sensações anormais (por exemplo, sensações de "lã de algodão")

> Gosto metálico

> Distorções da imagem corporal

, Sentimentos de irrealidade, despersonalização, desrealização

, Depressão, disforia, agorafobia

Adaptado de Brett J, et al. Management of benzodiazepine misuse and dependence. Aust Prescr. 2015;38:152-5.?

Na abordagem inicial de um indivíduo consumidor de benzodiazepinas devem ser verificados os critérios diagnósticos de dependência de uma substância, que podem ser consultados na Tabela 2 .

\section{COMO SUSPENDER?}

Na literatura, estão descritas diversas estratégias para a descontinuação de benzodiazepinas. É consensual que para que todo este processo se desenrole de forma segura e eficiente é necessário o envolvimento do utente
TABELA 2. Critérios de diagnóstico de dependência de substâncias de acordo com o CID-10.

Presença em simultâneo de 3 ou mais das seguintes condições, nos últimos 12 meses:

1. Desejo intenso (ou compulsivo) de tomar a substância

Dificuldade em controlar o consumo da substância, em termos de início, término ou níveis de consumo

Sintomatologia de abstinência aquando da sua interrupção ou

3. redução da dose, ou uso da substância, ou outra similar, com a intenção de alívio ou redução dos sintomas de abstinência

Evidência de tolerância, manifestada por aumento da dosagem

4. com o intuito de manter os mesmos efeitos conseguidos inicialmente com doses menores

Progressivo abandono e desinteresse por atividades

5. anteriormente agradáveis devido ao uso da substância,

ou aumento do tempo necessário para a sua aquisição ou recuperação dos seus efeitos

Persistência do uso de substância, apesar de evidência

6. de consequências nefastas, quer a nível cognitivo como comportamental

Adaptado de World Health Organization. Management of substance abuse: Dependence syndrome [homepage on the Internet]. [acedido em Jul 2019]. Disponível em: http://www.who.int/substance_abuse/terminology/definition1/en/. ${ }^{10}$

desde o início, nomeadamente pela sua capacitação com discussão de potenciais riscos e benefícios do uso desta medicação e negociação do plano de desmame. O indivíduo deve sentir-se como parte integrante do regime de descontinuação, devendo este ser flexível, mantendo-se motivado para controlar o ritmo e a duração do processo.

Deve ser alertado para o aparecimento de sintomas de abstinência, nomeadamente insónia e ansiedade, sendo de forma geral de intensidade moderada e de curta duração - dias a semanas. ${ }^{11}$

Aquando da ponderação da descontinuação destes fármacos deve ser confirmada a resolução da sintomatologia para a qual foram inicialmente prescritos. ${ }^{12}$

O elemento-chave do processo de desmame é a redução gradual, em semanas a meses, individualizada e adaptada a cada utente. Está recomendado o fornecimento do esquema de desmame, em formato escrito, para que o utente o possa consultar, assim como materiais de educação para a saúde, como panfletos que promovam a literacia sobre esta temática. ${ }^{12,13}$

Dentre os diversos esquemas de desmame existentes, a estratégia mais recomendada é a redução semanal de 25\% da dose a cada 2 a 3 semanas até à suspensão total. À medida que a dose é menor, a redução deverá ser mais gradual e, por isso, um esquema alternativo é redução mais lenta, de 12,5\%, após ser atingida metade da dose inicial. ${ }^{12,13}$ 
Um protocolo habitual de interrupção de benzodiazepinas durará entre 4 semanas a 6 meses, sendo tanto mais longo quanto maior a dependência. ${ }^{12}$

A conversão da benzodiazepina por uma dose equivalente de diazepam com posterior desmame é, por vezes, usada quando estamos perante benzodiazepinas de ação curta ou intermédia, em formulações que não permitem reduções da dose ou quando existe um elevado grau de dependência. ${ }^{12}$

Ao longo de todo este processo realça-se a importância da comunicação e da relação médico-doente, fundamental para garantir o sucesso da descontinuação e manter a motivação. Por outro lado, deve-se salientar a importância de resultados a longo prazo e não deixar que eventuais alarmismos (como por vezes instigados pelos meios de comunicação social) acelerem todo o processo. No relato de caso descrito, embora tenha sido fornecida informação escrita, a utente optou por uma suspensão abrupta da medicação, sinalizando a importância de garantir uma total compreensão e cumprimento da estratégia proposta.

A associação de terapia cognitivo-comportamental à descontinuação de benzodiazepinas revela uma melhoria das taxas de cessação, contudo essa diferença não se mantém a longo prazo. ${ }^{13}$

Na presença de comorbilidade, como antecedentes de abuso de substâncias ou doença psiquiátrica grave, não está aconselhada a descontinuação no âmbito dos cuidados de saúde primários, devendo ser referenciados para consulta de psiquiatria. ${ }^{12}$

\section{CONCLUSÃO}

Com este caso clínico, pretende-se consciencializar os profissionais de saúde para a importância e cuidados a ter na desprescrição de benzodiazepinas. Os médicos de família, como principais prescritores de benzodiazepinas, encontram-se numa posição privilegiada para a abordagem do uso crónico deste grupo farmacológico, assim como para o reconhecimento da dependência e das manifestações da síndrome de abstinência.

CONFLITOS DE INTERESSE: Os autores declaram não ter qualquer conflito de interesse na realização do presente trabalho.

FONTES DE FINANCIAMENTO: Não houve qualquer fonte de financiamento na realização do presente trabalho.
CONFIDENCIALIDADE DOS DADOS: Os autores declaram ter seguido os protocolos da sua instituição acerca da publicação dos dados de doentes.

CONSENTIMENTO: Consentimento do doente para publicação obtido.

PROVENIÊNCIA E REVISÃO POR PARES: Não comissionado; revisão externa por pares.

CONFLICTS OF INTEREST: The authors declare that they have no conflicts of interest.

FINANCIAL SUPPORT: This work has not received any contribution, grant or scholarship.

CONFIDENTIALITY OF DATA: The authors declare that they have followed the protocols of their work center on the publication of data from patients.

PATIENT CONSENT: Consent for publication was obtained.

PROVENANCE AND PEER REVIEW: Not commissioned; externally peer reviewed.

\section{REFERÊNCIAS}

1. Comissão de Farmácia e Terapêutica da Administração Regional de Saúde de Lisboa e Vale do Tejo. Boletim Terapêutico n 1/ 2017: Utilização de Benzodiazepinas: um grave problema de saúde pública. Lisboa: CFTARSLVT; 2017.

2. International Narcotics Control Board. Report of the International Narcotics Control Board for 2004. [acedido em Jul 2019]. Disponível em: https://www.incb.org/documents/Publications/AnnualReports/AR2004/AR_04_English.pdf

3. Starcevic $\vee$. Benzodiazepines for anxiety disorders: maximising the benefits and minimising the risks. Adv Psychiatr Treat. 2012;18:250-8.

4. Sateia MJ, Buysse DJ, Krystal AD, Neubauer DN, Heald JL. Clinical Practice Guideline for the Pharmacologic Treatment of Chronic Insomnia in Adults: An American Academy of Sleep Medicine Clinical Practice Guideline. J Clin Sleep Med. 2017;13:307-49. doi: 10.5664/jcsm.6470.

5. Aurora RN, Zak RS, Maganti RK, Auerbach SH, Casey KR, Chowdhuri $\mathrm{S}$, et al. Best practice guide for the treatment of REM sleep behavior disorder (RBD). J Clin Sleep Med. 2010; 6: 8595.

6. Direção-Geral da Saúde, Tratamento Sintomático da Ansiedade e Insónia com Benzodiazepinas e Fármacos Análogos: Norma no 055/2011, de 27/10/2011, atualizada a 21/01/2015. Lisboa: DGS; 2015.

7. Prescribing drugs of dependence in general practice, Part B - Benzodiazepines [e-book]. Melbourne: The Royal Australian College of General Practitioners [acedido em Jul 2019]. Disponível em: https://www.racgp.org.au/FSDEDEV/media/ documents/Clinical\%20Resources/Guidelines/Drugs\%20 of\%20dependence/Prescribing-drugs-of-dependence-in-general-practice-Part-B-Benzodiazepines.pdf.

8. Liebrenz M, Gehring M-T, Buadze A, Caflisch C. High-dose benzodiazepine dependence: a qualitative study of patients' perception on cessation and withdrawal. BMC Psychiatry. 2015; 15: 116. doi: 10.1186/s12888-015-0493-y. 
9. Brett J, Murnion B. Management of benzodiazepine misuse and dependence. Aust Prescr. 2015;38:152-5.

10. World Health Organization. Management of substance abuse: Dependence syndrome [homepage on the Internet]. [acedido em Jul 2019]. Disponível em: http://www.who.int/substance_ abuse/terminology/definition1/en/.

11. Quelle place pour les benzodiazépines dans l'insomnie? Recommandation sur le bon usage du médicament. Paris: HAS; 2015.

12. Comissão de Farmácia e Terapêutica da Administração Regional de Saúde de Lisboa e Vale do Tejo. Anexo I ao Boletim Terapêutico N 1/2017. Estratégias para a Descontinuação de Benzodiazepinas. Lisboa: CFTARSLVT; 2017.

13. Pottie K, Thompson W, Davies S, Grenier J, Sadowski CA, Welch $\mathrm{V}$, et al. Deprescribing benzodiazepine receptor agonists. Can Fam Physician. 2018;64:339-51. 